\title{
Association between diet quality and obesity indicators among the working-age adults in Inner Mongolia, Northern China: a cross-sectional study
}

Lu Jia ${ }^{1+}$, Haiwen $\mathrm{Lu}^{2+}$, Jing $\mathrm{Wu}^{3}$, Xuemei Wang ${ }^{1 *}$ (D), Wenrui Wang ${ }^{4}$, Maolin Du', Peiyu Wang ${ }^{5}$, Sha Du', Yuenan $\mathrm{Su}^{1}$ and Nan Zhang ${ }^{6}$

\begin{abstract}
Background: Obesity is a major risk factor for the global burden of disease in countries that are economically developed or not. This study aimed to investigate the association between diet quality and obesity indicators applying DASH and aMed.

Methods: This cross-sectional study on adult nutrition and chronic disease in Inner Mongolia $(n=1320)$. Dietary data were collected using 24-h diet recall for 3 consecutive days and weighing method. DASH and aMed were used to assess the dietary quality. WC, BMI and WC-BMI were used as obesity indicators. Logistic regression models were used to examine the associations between diet quality and obesity indicators.

Results: Higher diet quality, assessed by DASH, was only associated with WC. The odds ratio (OR) for abdominal obesity in the highest tertile of DASH scores compared with the lowest was 0.71 ( $95 \%$ confidence interval (CI) 0.53 , 0.96 ; $P_{\text {trend }}=0.03$ ). Furthermore, aMed was inversely associated with obesity indicators. OR for abdominal obesity in the highest tertile of aMed score compared with the lowest were $0.63\left(95 \% \mathrm{Cl} 0.47,0.87 ; P_{\text {trend }}=0.005\right)$ and 0.57 $\left(95 \% \mathrm{Cl} 0.41,0.77 ; P_{\text {trend }}=0.02\right)$ for overweight and obesity, respectively, and $0.60\left(95 \% C l 0.44,0.81 ; P_{\text {trend }}=0.02\right)$ for high obesity risk.
\end{abstract}

Conclusions: Our findings suggest that dietary quality assessed using aMed is more closely associated with obesity than assessment using DASH in working-age adults in Inner Mongolia. The Mediterranean diet can be recommended as a healthy diet to control weight.

Keywords: Diet quality, Obesity, Working-age population, DASH diet, Mediterranean diet

\footnotetext{
* Correspondence: wangxm_zsu@163.com

${ }^{+}$Lu Jia and Haiwen Lu contributed equally to this work.

'Department of Health Statistics, School of Public Health, Inner Mongolia Medical University, Hohhot 010110, China

Full list of author information is available at the end of the article
}

(C) The Author(s). 2020 Open Access This article is licensed under a Creative Commons Attribution 4.0 International License, which permits use, sharing, adaptation, distribution and reproduction in any medium or format, as long as you give appropriate credit to the original author(s) and the source, provide a link to the Creative Commons licence, and indicate if changes were made. The images or other third party material in this article are included in the article's Creative Commons licence, unless indicated otherwise in a credit line to the material. If material is not included in the article's Creative Commons licence and your intended use is not permitted by statutory regulation or exceeds the permitted use, you will need to obtain permission directly from the copyright holder. To view a copy of this licence, visit http://creativecommons.org/licenses/by/4.0/ The Creative Commons Public Domain Dedication waiver (http://creativecommons.org/publicdomain/zero/1.0/) applies to the data made available in this article, unless otherwise stated in a credit line to the data. 


\section{Background}

Obesity is a worldwide public health problem, with increased risks of cardiovascular and cerebrovascular diseases, diabetes, and probability of death [1]. Body mass index (BMI) and waist circumference (WC) are common obesity indicators. BMI is more closely related to general obesity, and WC is more closely related to abdominal obesity and metabolic related diseases [2]. In 2017 Global Burden of Disease (GBD) study [3], high body mass index (BMI) was ranked fourth among mortality risk factors. As obesity indicator, BMI-WC was a weight-related risk outcome combined WC and BMI. A Canadian study examined the association between occupational sedentary behavior and BMI-WC, and found that those at very high risk and extremely high risk were associated with increasing occupational sedentary time compared to the lowest obesity risk population among men [4]. Great changes have taken place in the spectrum of disease as well as the age and occupational structure of the patient population in China over the past few decades [5]. The working-age adults are at high risk for obesity, and the prevalence of overweight and obese have increased rapidly [6]. People who work long hours have irregular lifestyle and have little time to exercise, further promoting weight gain. Unbalanced consumption of foods high in energy and low in essential nutrients contributes to overweight or obesity among working-age adults, which has a further negative impact on health. If obesity is effectively controlled during this period, in the long run, obesity complications such as diabetes and stroke might be prevented, resulting in lower incidence of obesity complications at older ages and improved quality of life among the population.

Obesity is a complex metabolic disease that is related to multiple factors. It is believed to result from a combination of genetic, environmental, and social factors, among which consumption of an unhealthy diet plays an important role [7]. The current obesity epidemic is attributed to excessive energy intake and unbalanced energy intake. A high-quality diet is important for the prevention and treatment of a variety of chronic diseases including obesity, cancer, cardiovascular disease, and diabetes [8].

The Dietary Approaches to Stop Hypertension (DASH) and Mediterranean diets have been recognised as high quality diets worldwide. The DASH aimed to prevent and control high blood pressure by intaking reasonable diets. Studies have shown that the DASH diet has beneficial effects on obesity, hypertension, dyslipidemia, diabetes, gestational diabetes, and cardiovascular disease [9]. The Mediterranean diet is predominantly plant-based, rich in vegetables, fruit, nuts, legumes, fish or seafood, with low consumption of red meat and sweets and moderate intake of wine and dairy. Studies have proven that the Mediterranean diet can help to protect the heart and brain, prevent diabetes, prolong life, and reduce the risks of Parkinson disease, Alzheimer disease, and stroke [10]. One approach to assessing overall diet quality is to use a priori scores, based on dietary recommendations, such as DASH and alternate Mediterranean diet (aMed) scores. The quality of the DASH diet is quantified using DASH scores, and the aMed scores can reflect adherence to the Mediterranean diet. Many studies have confirmed that DASH and aMed scores reflect well the respective dietary quality [11].

Diets differ among different populations. Inner Mongolia is inland region, located in northwest China, and the population has dietary cultures and ethnic characteristics that differ from the rest of China. People in Inner Mongolia consume less vegetables and fruits, and more meat, oil and salt [12]. Inner Mongolia consists mainly of Han and Mongolian. Compared with Han population, the dietary pattern of Mongolians tends to be rich in whole milk, fats and oils, which might increase greater risk of obesity in Mongolia [13]. The prevalence of obesity in Inner Mongolia is relatively serious. Studies have shown that the prevalence of overweight and obesity in Inner Mongolia was 37.19 and $18.44 \%$ in 2014 [14], which was much higher than the national level [15] and higher than the global level [16]. Despite the heavy burden of obesity in the region, no current and comprehensive estimates of the obesity epidemic exist among working-age people in this part of China.

The main objective of this study was to assess the dietary quality of working-age people in Inner Mongolia using DASH and aMed scores. We also aimed to explore the relationship between dietary quality and obesity indicators using obesity as an entry point to provide a reasonable and healthy diet for the prevention of obesity, thereby reducing the incidence of obesity caused by dietary factors.

\section{Methods}

\section{Study design and subjects}

This cross-sectional study was a surveillance survey of chronic disease and nutrition in Chinese adults in Inner Mongolia conducted in 2015. The study design and questionnaire used have previously been described [12]. The surveillance survey consisted of dietary and non-dietary investigation, including information of sociodemographic characteristics, health behaviors, personal and familial medical history, and dietary information. Multi-stage cluster random sampling method was used to select a representative sample of residents aged 18 years and older. We selected the age range between 18 to 60 years as the workingage adults in this study. 


\section{Diet data collection}

The 24-h diet recall for 3 consecutive days and weighing method were used for the dietary investigation. The 24$\mathrm{h}$ diet recall for 3 consecutive days, which is a dietary survey method recommended by the Chinese Dietary Guidelines, and aims to know about the residents' intake of nutrients and foods. In the 24-h recall, every participant reported the type and amount of all foods (including alcohol) consumed in the past $24 \mathrm{~h}$ for 3 consecutive days. Information of condiments such as salt and soy sauce, and cooking oils consumption was collected with weighing method, in which changes in the household food inventory were measured. The average daily intake of nutrients and total energy were calculated according to the Chinese Food Composition Table, 2007 [17].

\section{Diet quality indices}

DASH and aMed scores were used to assess the dietary quality of study participants. We calculated the DASH scores using the method described by Fung [18] et al. to determine adherence to the DASH diet, which consists of eight components. The scoring system is based on quintile rankings; for intakes of fruit; vegetables (excluding potatoes); low-fat dairy products; whole grains; and nuts, seeds, and legumes. Individuals receive a score from 1 (lowest quintile) to 5 (highest quintile). In contrast, individuals receive a score from 1 (highest quintile) to 5 (lowest quintile) for intakes of sodium, sugarsweetened beverages, and red and processed meat. Male and female are classified into quintiles separately. A higher DASH score indicates greater compliance with the DASH diet, and therefore, a higher diet quality.

The aMed scores were developed by Fung [19] et al. to assess the quality of the Mediterranean diet. For foods and nutrients related to a beneficial health outcome, 1 point for each item is assigned to subjects with high intake of whole grains, vegetables, fruit, legumes, nuts, fish, and the ratio of monounsaturated to saturated fat, based on the sex-specific median of the study population; all other intakes receive 0 points. People with red and processed meat consumption below the median receive 1 point, and those whose consumption is at or above the median are assigned 0 points. For alcohol, 1 point is given for consumption between 5 and $25 \mathrm{~g}$ per day. The possible score range for aMed is 0 to 9 , with higher scores indicating closer adherence to the Mediterranean diet.

\section{Anthropometric measurements}

Height, weight, and waist circumference (WC) were directly measured by trained and evaluated health workers, using standard methods. Height and weight were measured once. WC was measured twice, and the mean value was used for the analyses.

\section{Definition of obesity}

WC, BMI, and WC-BMI were used as indicators of obesity. Based on the World Health Organization recommendations for Asian adults, abdominal obesity was defined as $W C \geq 85 \mathrm{~cm}$ for female and $\geq 90 \mathrm{~cm}$ for male. BMI was calculated by dividing weight $(\mathrm{kg})$ by the square of height $\left(\mathrm{m}^{2}\right)$. According to the Chinese definitions [20], BMI was classified into two categories: underweight or normal weight $\left(<24 \mathrm{~kg} / \mathrm{m}^{2}\right)$, and overweight and obese $\left(\geq 24 \mathrm{~kg} / \mathrm{m}^{2}\right)$. We combined WC and BMI as another weight-related risk outcome. BMI-WC is divided into low obesity risk and high obesity risk. Low risk individuals included those who had both BMI $<24$ $\mathrm{kg} / \mathrm{m}^{2}$ and $\mathrm{WC}<90 \mathrm{~cm}$ (male) or $<85 \mathrm{~cm}$ (female); high risk participants included those with BMI $\geq 24 \mathrm{~kg} / \mathrm{m}^{2}$ or $W C \geq 90 \mathrm{~cm}$ (male) or $\geq 85 \mathrm{~cm}$ (female).

\section{Other variables}

Each participant completed a questionnaire about sociodemographic characteristics, health behaviors, and personal and familial medical history. Sociodemographic characteristics included gender, age, place of residence, ethnicity, and education level. Health behaviors included smoking status, drinking status, physical activity, and meals eaten outside the home. Personal and familial medical history include chronic diseases such as hypertension and diabetes mellitus.

\section{Statistical methods}

Continuous variables following normal distribution were expressed as mean \pm standard deviation $(S D)$, and variables not following normal distribution were expressed as median (range, $P_{25}$ to $P_{75}$ ) and categorical variables were expressed as number (percentage). Participants were categorized based on tertiles of DASH scores and aMed scores. We used one-way analysis of variance or nonparametric test for continuous variables and chisquare test for categorical variables. Associations between diet quality and obesity indicators were examined using logistic regression models. In Model 1, we did not adjust for any factors; Model 2 was adjusted for sex, age, place of residence, ethnicity, education level, smoking status, drinking status, physical activity, meals eaten outside the home, family history of chronic diseases, hypertension, diabetes, and energy intake. A $P$-value $<0.05$ (two-tailed) was used to indicate statistical significance. All statistical analyses were performed with IBM SPSS software version 19.0 (IBM Corp, Armonk, NY, USA).

\section{Results}

\section{Study participant characteristics}

The present study included 1320 working-age people aged 18 to 60 years. The characteristics of study participants across tertiles of diet quality indices are presented 
in Table 1. As shown in the table, most participants in the highest tertile of diet quality indices lived in urban, were Han ethnicity, and had higher education levels, sufficient physical activity, a family history of chronic diseases, and meals eaten outside the home (all $P<0.05$ ).

\section{Outcome data}

In total, 498 (38.5\%) participants had abdominal obesity, 784 (60.6\%) were overweight and obesity, and 816 (63.1\%) were at high risk of obesity. After standardization using $2010 \mathrm{Na}-$ tional Census Data, the standardized prevalence rates of abdominal obesity, overweight and obesity, and high risk of obesity were $34.5,56.8$, and $58.6 \%$, respectively (Table 1 ).

\section{Food group and nutrients intakes}

Compared with participants who had lower DASH scores, those with higher DASH scores had higher intakes of all foods except alcohol, red meat and processed meat, and sweetened beverages, as well as lower intakes of fat, total fatty acids, saturated fatty acids, monounsaturated fatty acids, and sodium $\left(P_{\text {trend }}<0.001\right)$. Individuals in the third tertile of aMed scores had significantly higher food intakes except for alcohol, red and processed meat, low-fat dairy, and sweetened beverages $\left(P_{\text {trend }}<0.001\right)$, as compared with participants in the first tertile (Table 2).

Table 1 Characteristics of the subjects by DASH and aMed score groups ${ }^{a}$

\begin{tabular}{|c|c|c|c|c|c|c|c|c|c|c|}
\hline & & \multirow[t]{2}{*}{ Total } & \multicolumn{4}{|l|}{ DASH } & \multicolumn{4}{|l|}{ aMed } \\
\hline & & & $\mathrm{T} 1$ & $\mathrm{~T} 2$ & T3 & $P$ & $\mathrm{~T} 1$ & $\mathrm{~T} 2$ & $\mathrm{~T} 3$ & $P$ \\
\hline WC & Abdominal obesity ${ }^{a}$ & $498(38.5)$ & 184(40.9) & 151(39.7) & 163(35.1) & 0.17 & 167(39.4) & 194(42.7) & 137(32.9) & 0.01 \\
\hline $\mathrm{BMl}$ & Overweight and obesity ${ }^{a}$ & $784(60.6)$ & 278(61.8) & $236(62.1)$ & $270(58.2)$ & 0.42 & $276(65.1)$ & $273(60.1)$ & $235(56.5)$ & 0.04 \\
\hline WC-BMI & High risk ${ }^{a}$ & $816(63.1)$ & $286(63.6)$ & $248(65.3)$ & $282(60.8)$ & 0.39 & $285(67.2)$ & $286(6)$ & 245(58.9) & 0.04 \\
\hline Gender & Female & 699(53.0) & 228(49.9) & 204(52.4) & $267(56.3)$ & 0.14 & $230(53.5)$ & $233(50.5)$ & $236(55.0)$ & 0.40 \\
\hline \multirow[t]{4}{*}{ Age } & $<35$ & 190(14.4) & $51(11.1)$ & $64(16.5)$ & $75(15.8)$ & 0.02 & $65(15.1)$ & $56(12.2)$ & 69(16.1) & 0.002 \\
\hline & $35-44$ & $316(23.9)$ & $130(28.5)$ & $72(18.5)$ & $114(24.1)$ & & 123(28.6) & $95(20.6)$ & $98(22.8)$ & \\
\hline & $45-54$ & $535(40.5)$ & 176(38.5) & 168(43.2) & 191(40.3) & & $160(37.2)$ & 188(40.8) & 187(43.6) & \\
\hline & $55-60$ & $279(21.1)$ & $100(21.9)$ & $85(21.8)$ & $94(19.8)$ & & $82(19.1)$ & $122(26.4)$ & $75(17.5)$ & \\
\hline Place of residence & Urban & $527(39.9)$ & $122(26.7)$ & $127(32.7)$ & 278(58.7) & $<0.001$ & 128(29.8) & $151(32.8)$ & $248(57.8)$ & $<0.001$ \\
\hline \multirow[t]{3}{*}{ Ethnicity } & Han & 1034(78.3) & $322(70.5)$ & $308(79.2)$ & $404(85.2)$ & $<0.001$ & $321(74.6)$ & $362(78.5)$ & $351(81.8)$ & $<0.001$ \\
\hline & Mongolian & $233(17.7)$ & 123(26.9) & $65(16.7)$ & $45(9.5)$ & & $97(22.6)$ & $88(19.1)$ & $48(11.2)$ & \\
\hline & Other minority & $53(4.0)$ & $12(2.6)$ & $16(4.1)$ & $25(5.3)$ & & $12(2.8)$ & $11(2.4)$ & $30(7.0)$ & \\
\hline \multirow[t]{3}{*}{ Education level } & Low & $476(36.1)$ & $227(49.7)$ & 140(36.0) & 109(23.0) & $<0.001$ & $201(46.7)$ & 184(39.9) & $91(21.2)$ & $<0.001$ \\
\hline & Medium & $485(36.7)$ & 157(34.3) & 147(37.8) & 181(38.2) & & 149(34.7) & 162(35.1) & 174(40.6) & \\
\hline & High & $359(27.2)$ & 73(16.0) & $102(26.2)$ & 184(38.8) & & 80(18.6) & $115(25.0)$ & 164(38.2) & \\
\hline \multirow[t]{3}{*}{ Smoking status } & Current smokeing & 409(31.0) & 157(34.4) & 117(30.1) & 135(28.5) & 0.40 & $141(32.8)$ & 150(32.6) & $118(27.5)$ & 0.33 \\
\hline & Quitting smoking & $55(4.2)$ & $17(3.7)$ & $17(4.4)$ & $21(4.4)$ & & $15(3.5)$ & 18(3.9) & $22(5.1)$ & \\
\hline & not smoking & $856(64.8)$ & 283(61.9) & $255(65.5)$ & $318(67.1)$ & & 274(63.7) & 293(63.5) & 289(67.4) & \\
\hline \multirow[t]{3}{*}{ Physical activity } & None & 100(7.6) & $43(9.4)$ & $38(9.8)$ & $19(4.0)$ & $<0.001$ & $37(8.6)$ & $41(8.9)$ & $22(5.1)$ & 0.001 \\
\hline & Inadequate & $376(28.5)$ & $99(21.7)$ & 113(29.0) & 164(34.6) & & $110(25.6)$ & $112(24.3)$ & 154(35.9) & \\
\hline & Sufficient & $844(63.9)$ & $315(68.9)$ & $238(61.2)$ & $291(61.4)$ & & 283(65.8) & $308(66.8)$ & 253(59.0) & \\
\hline $\begin{array}{l}\text { Meals eaten } \\
\text { outside the home }\end{array}$ & Yes & 230(17.4) & $62(13.6)$ & 78(20.1) & $90(19.0)$ & 0.03 & $58(13.5)$ & $90(19.5)$ & $82(19.1)$ & 0.03 \\
\hline \multirow[t]{3}{*}{ Drinking status } & Never & $802(60.8)$ & $275(60.2)$ & $240(61.7)$ & $287(60.6)$ & 0.12 & $270(62.8)$ & $266(57.7)$ & $266(62.0)$ & 0.18 \\
\hline & Moderate & $367(27.8)$ & $126(27.6)$ & $96(24.7)$ & 145(30.6) & & $119(27.7)$ & $129(28.0)$ & $119(27.7)$ & \\
\hline & Excess & 151(11.4) & $56(12.2)$ & 53(13.6) & $42(8.6)$ & & $41(9.5)$ & $66(14.3)$ & $44(10.3)$ & \\
\hline Family history & Yes & $771(58.4)$ & 243(53.2) & $222(57.1)$ & $306(64.6)$ & 0.002 & $224(52.1)$ & $261(56.6)$ & $286(66.7)$ & $<0.001$ \\
\hline Hypertension & Yes & $552(41.8)$ & 195(42.7) & 169(43.4) & 188(39.7) & 0.48 & 173(40.2) & 194(42.1) & 185(43.1) & 0.69 \\
\hline Diabetes & Yes & $84(6.4)$ & $26(5.7)$ & $25(6.4)$ & $33(7.0)$ & 0.73 & $24(5.6)$ & $36(7.8)$ & $24(5.6)$ & 0.29 \\
\hline
\end{tabular}

Variables are presented as frequency (percentage) (\%)

${ }^{a}$ After standardization using the 2010 National Census Data, the standardized prevalence rates of abdominal obesity, overweight obesity, and high risk of obesity were $34.5,56.8$, and $58.6 \%$, respectively 


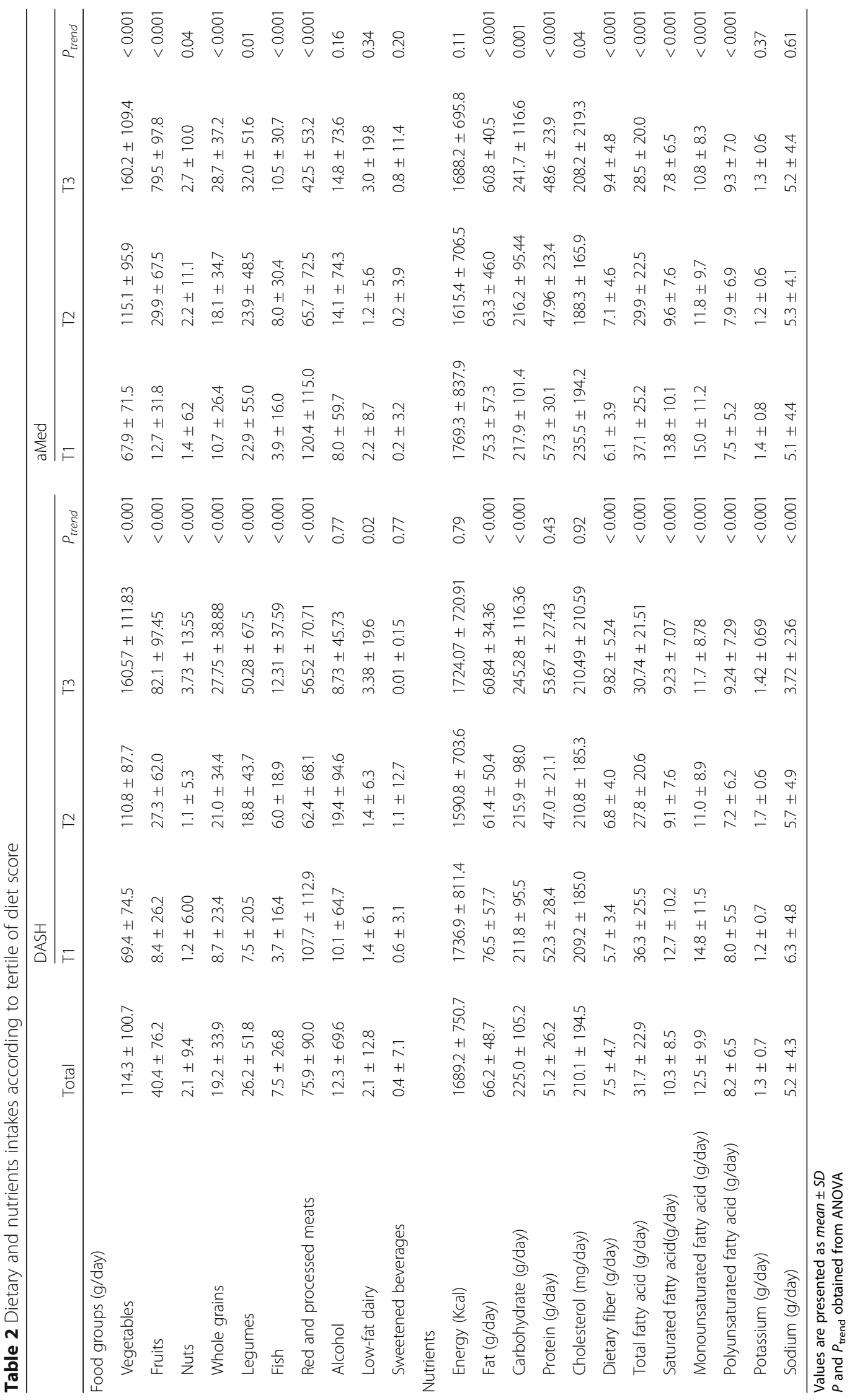




\section{Association between diet quality scores and obesity indicators}

In univariate analyses, there was no significant association between dietary quality, as assessed using DASH scores, and obesity indicators. After adjustment for confounding factors, higher diet quality assessed using DASH was only associated with WC. The odds ratio $(O R)$ for abdominal obesity in the highest tertile of DASH scores, compared with the lowest, was 0.71 (95\% confidence interval $\left.(C I) 0.53,0.96 ; P_{\text {trend }}=0.03\right)$. Furthermore, aMed was inversely associated with obesity indicators. After adjusting for confounding factors, OR for abdominal obesity in the highest tertile of a aMed scores, compared with the lowest were 0.63 (95\% CI $\left.0.47,0.87 ; P_{\text {trend }}=0.005\right)$ and 0.57 (95\% CI 0.41, 0.77; $\left.P_{\text {trend }}=0.02\right)$ for overweight and obesity, respectively, and $0.60\left(95 \%\right.$ CI $\left.0.44,0.81 ; P_{\text {trend }}=0.02\right)$ for high obesity risk. Confounding factors included gender, age, place of residence, ethnicity, education level, smoking status, drinking status, physical activity, meals eaten outside the home, family history of chronic diseases, hypertension, diabetes, and energy intake, which may influence the results when assessing the relationship between diet and obesity indicators (Table 3 ).

\section{Discussion}

In this cross-sectional study, we evaluated the diet quality of the working-age adults in Inner Mongolia in 2015 and its association with obesity indicators. Overweight

Table 3 Logistics regression model of diet quality scores and obesity indicators

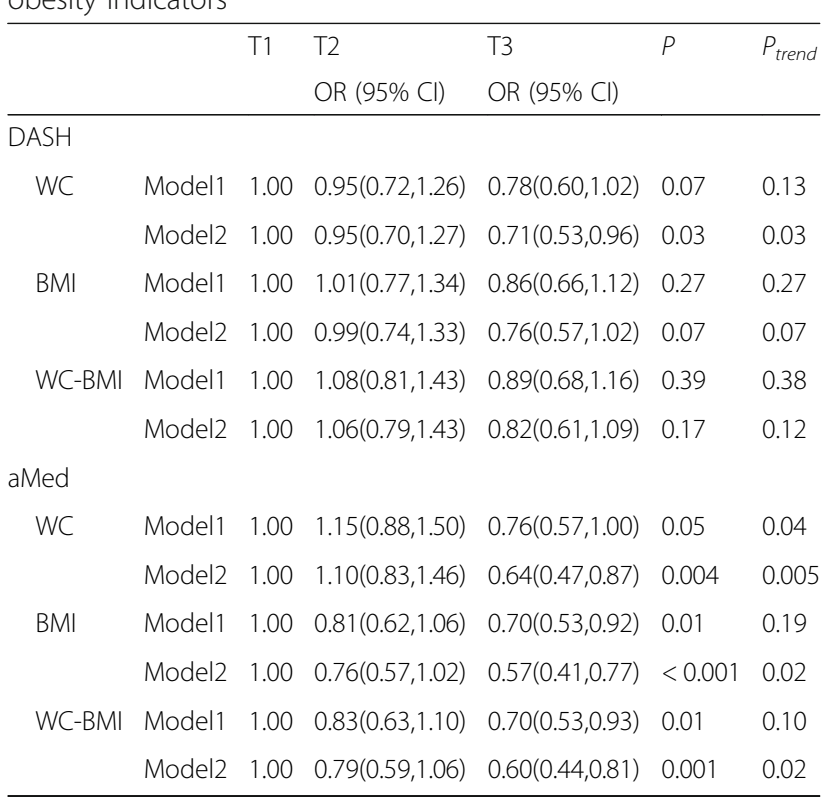

Model 1: crude data;

Model 2: Adjusted for gender, age, place of residence, ethnicity, education level, smoking status, drinking status, physical activity, meals eaten outside the home, family history of chronic diseases, hypertension, diabetes and energy intake. and obesity are common among the working-age adults. The standardized prevalence of abdominal obesity, overweight and obesity, and high obesity risk were 34.5, 56.8, and $58.6 \%$, respectively. In the same year, the rate of overweight and obesity among Chinese adults over 18 years old was $42.6 \%$ [21]. The prevalence of overweight and obesity was 38.6 and $18.4 \%$, respectively, among the working-age adults in Spain [22]. As a developing country, the prevalence of overweight among Malaysian adults was $51.2 \%$ [23]. In 2014, the prevalence rate of overweight and obesity in 20 European countries reached $53.1 \%$ [24].

Some studies $[25,26]$ in the Mediterranean region about Mediterranean Diet are using MEDAS to determine the level of adherence to the Mediterranean diet. However, MEDAS is not very suitable for the nonMediterranean region. Therefore, we used DASH and aMed scores to assess the dietary quality of working-age adults in Inner Mongolia. The overall dietary quality score of the working-age adults in Inner Mongolia was lower and the dietary quality was poor. The median aMed score for participants was only 3(range, 2 to 4), and the median DASH score was 21(range, 19 to 24) (Table S1). A case-control study in China showed that the DASH score of residents in Hong Kong was 22.6 [27]; another case-control study showed that the aMed score for residents of Guangzhou, China was 3.92 [28]. To our knowledge, this is the first observational study investigating the association between obesity indicators and diet quality, as assessed by DASH and aMed, among the working-age adults of Inner Mongolia. A metaanalysis showed that the Mediterranean diet was associated with a reduced risk of metabolic syndrome and all components, including abdominal obesity [29]. In a randomized controlled clinical trial, after 3 months of dietary intervention, Azadi-Yazdi found that the participants following DASH diet had reduced BMI [30]. The results of an interventional study showed that adherence to the Mediterranean diet was associated with lower body weight and lower waist circumference [31]. Consistent with other findings [26-28], our study showed that the DASH score was inversely associated with WC whereas the aMed dietary score was negatively correlated with WC, BMI, and WC-BMI. These results indicated that the Mediterranean diet is more closely related to obesity compared with the DASH diet in our population.

The intake of red and processed meats among our participants with higher aMed scores was much lower than in those with higher DASH scores. As an important part of both the DASH diets and Mediterranean diets, red and processed meats are rich in protein, cholesterol, and saturated fatty acids, which provide greater energy; there is a significant positive correlation between energy intake and obesity [32]. Epidemiological studies have indicated 
that the consumption of red meat is positively correlated with general and abdominal obesity [33]. According to reports, adhering to a meat diet model for 5 years increases the risk of general obesity [34]. A national crosssectional study in the United States reported that those who consumed more meat had a much higher daily total energy intake, and red meat was positively associated with central obesity [35]. Furthermore, a study conducted in the Chinese population showed that intake of total red meat, intake of fresh red meat, and intake of fatty fresh red meat were positively associated with an increased WC measurement (men only) and risk of abdominal obesity among Chinese adults aged 18 to 75 [36]. These studies suggest the role that red and processed meats plays in obesity.

Working-age participants with a higher-quality diet, as assessed using aMed scores, had lower energy intake than those with high dietary quality, assessed using DASH scores. The fundamental cause of obesity is an energy imbalance between calories consumed and calories expended [37]. Epidemiological studies in Europe and the United States have found a significant positive correlation between energy intake and obesity-related indicators such as weight gain, BMI, and WC [38]. A recent meta-analysis showed that limiting the intake of energy can promote weight loss and improve metabolism [39].

People with higher dietary quality assessed by DASH had higher consumption of total fatty acids and saturated fatty acids than those with higher dietary quality assessed by aMed. However, a growing body of evidence suggests that the level of saturated fatty acid intake is positively correlated with the occurrence of cardiovascular and cerebrovascular diseases [40]. A 4-week experimental study among overweight or obese men showed that substituting dietary saturated fat with unsaturated fat can induce a significant loss of body weight and fat mass without a significant change in total energy or fat intake [41]. Saturated fatty acids in the diet can reduce leptin deficiency or leptin resistance and induce body obesity [42]. This may also be the reason why aMed was more closely related to obesity indicators than DASH in our population.

The DASH considers the component of sweetened beverages separately, with a recommendation of five servings or less per week [43]. Lower intake will affect the dietary quality of DASH evaluation. However, consumption of sweetened beverages in our population was very low, with average daily intake only $0.37 \mathrm{~g}$, which is far below the standard intake. And sweetened beverage is not one of items of aMed. Therefore, sweetened beverages are a factor that affects the relationship between diet quality and obesity. It is well known that sweetened beverages are harmful to metabolism. A recent study published in Diabetes Care found that body weight increased more among those who increased their sugary beverage consumption than among those who maintained a stable consumption or decreased their consumption of sweetened beverages [44]. Sweetened beverages have a lower degree of satiety than solid foods containing the same amount of calories and their consumption stimulates appetite, which may lead to greater calorie intake [45]. Therefore, when using DASH to assess the dietary quality among our population, DASH scores only reflected the sweetened beverage intake of some people, which represents only a small part of the cause of obesity. This may be the reason why aMed is more suitable for evaluating the relationship between obesity and diet quality than DASH.

In our study, cholesterol intake gradually decreased with higher diet quality, according to the Mediterranean diet. Interestingly, compared with higher aMed scores, the intake of cholesterol was higher in participants with higher DASH scores, and the intake of cholesterol increased gradually with increased dietary quality. A cohort study published in JAMA recently showed that higher consumption of dietary cholesterol was significantly associated with higher risk of incident cardiovascular disease and all-cause mortality [46]. Therefore, our results showed that aMed is more able to reflect the quality of the diet, it is suitable for evaluating the dietary quality of the Inner Mongolian population compared with DASH.

After analyzing the relationship between food components and obesity indicators according to the DASH and aMed indices, we found that people with high quality scores in the DASH diet and Mediterranean diet had relatively high scores for fruits, vegetables, whole grains, nuts and legumes, and red and processed meats. These results indicate that the composition of these two dietary patterns have many similarities. Moreover, the DASH and Mediterranean diets also differ in many respects. DASH is known as Dietary Approaches to Stop Hypertension, which considers sodium intake separately. A large number of studies have shown that potassium and sodium are associated with hypertension, the prognosis of chronic kidney disease, and cardiovascular events. Compared with participants who had higher dietary quality according to aMed, the intake of potassium was higher and sodium was lower in those with higher DASH dietary quality scores in our study. So comprehensively, DASH is suitable for assessing the relationship between dietary quality and certain diseases such as hypertension, and aMed is more suitable for assessing the relationship between dietary quality and obesity.

In addition to being a tool for preventing obesity, growing evidence shows that the Mediterranean diet is associated with diabetes, hypertensive, metabolic 
syndrome and other diseases [47]. The Mediterranean diet demonstrated to exert a preventive effect toward cardiovascular diseases, in both Mediterranean and nonMediterranean populations [47, 48]. Some studies also suggested a potential role in preventing certain cancers [49]. Newer research has showed that higher adherence to the Mediterranean diet was associated with lower risk of cognitive decline, depression, and other mental disorders [50]. Therefore, the Mediterranean diet could be used in public health nutrition policies in order to promote health.

\section{Limitations}

There are several limitations to this study. First, the study design was cross-sectional, the associations are not proof of causality, and reverse causality bias could be present. Additional cohort studies with follow-up data are necessary to strengthen the understanding of the associations identified here. Secondly, our research was conducted in Inner Mongolia, which investigated the local dietary and evaluated the relationship between dietary quality and obesity. Different from other parts of China, the diet in Inner Mongolia residents is characterized by less intake of vegetables, fruits and fish, and more intake of meat. Our conclusion may be applicable to those regions whose diet characteristics are similar with diets in Inner Mongolia. At the same time, our research population was obtained by the Multi-stage cluster random sampling method, and the samples are well representative. Thirdly, a $24-\mathrm{h}$ recall and weighing method were used for the dietary investigation, which may cause recall bias. Considering the limitations of study, our results need to be confirmed in further studies, especially for prospective studies in Inner Mongolia.

\section{Strengths}

Our study population chose working age population. If obesity is effectively controlled in the working age population, it can prevent diseases such as diabetes and stroke, thereby reducing the incidence of obesity complications in the elderly and improving people's quality of life. We evaluated the relationship between diet and obesity, and finally found that aMed is more suitable for evaluating the relationship between diet quality and obesity in Inner Mongolia, and we can prevent obesity by the Mediterranean diet.

\section{Conclusions}

A higher diet quality, as assessed using aMed scores, was more closely associated with obesity among working-age people in Inner Mongolia as compared with assessment using DASH scores. The Mediterranean diet can be recommended for underdeveloped regions as a healthy diet to control weight.

\section{Supplementary information}

Supplementary information accompanies this paper at https://doi.org/10. 1186/s12889-020-09281-5.

Additional file 1: Table S1. Characteristics Total and component scores of the diet quality indices

\section{Abbreviations}

95\% Cl: 95\% Confidence interval; BMI: Body mass index; OR: Odds ratio; DASH: The Dietary Approaches to Stop Hypertension:; aMed: Alternate Mediterranean diet; WC: Waist circumference; SD: Standard deviation

\section{Acknowledgements \\ None.}

\section{Authors' contributions}

Conceptualization, $L$ and XMW; Data curation, JW, WRW, MLD, SD, YNS; Formal analysis, $L J$ and HWL; Funding acquisition, $L J$ and XMW;

Methodology, PYW; Supervision, XMW; Writing - original draft, LJ and HWL; Writing - review \& editing, LJ, NZ. All authors have read and approved the final version to be submitted for consideration for publication.

\section{Funding}

This work was supported by Natural Science Foundation of Inner Mongolia, grant number 2019MS08112, Inner Mongolia Science and Technology Project (Study on Epidemiological Characteristics and Its Key Techniques for Prevention and Control of Brucellosis and Q fever), and Research Innovation Subsidy Project for Graduate Students of Inner Mongolia, grant number S2018112013Z. These funding played roles in the analysis, and interpretation of data and in writing the manuscript in this study.

\section{Availability of data and materials}

The full data set are available from the corresponding author under reasonable request.

\section{Ethics approval and consent to participate}

The survey was approved by the Ethical Committee of the National Institute for Nutrition and Food Safety, Chinese Center for Disease Control and Prevention. All participants provided written informed consent before the start of the investigation.

\section{Consent for publication}

Not Applicable.

\section{Competing interests}

The authors declare that they have no competing interests.

\section{Author details}

${ }^{1}$ Department of Health Statistics, School of Public Health, Inner Mongolia Medical University, Hohhot 010110, China. ²Department of Medical Imaging, Affiliated Hospital of Inner Mongolia Medical University, Inner Mongolia Medical University, Hohhot 010050, China. ${ }^{3}$ National Center for Chronic and Non-Communicable Disease Control and Prevention, Chinese Center for Disease Control and Prevention, Beijing 100050, China. ${ }^{4}$ Department of Chronic Disease Control and Prevention, Inner Mongolia Center for Disease Control and Prevention, Hohhot 010031, China. ${ }^{5}$ Department of Social Medicine and Health Education, School of Public Health, Peking University Health Science Center, Beijing 100191, China. ${ }^{6}$ Department of Hygienic Toxicology, School of Public Health, Inner Mongolia Medical University, Hohhot 010110, China.

Received: 29 February 2020 Accepted: 20 July 2020

Published online: 25 July 2020

\section{References}

1. Jackson CL, Yeh HC, Szklo M, et al. Body-mass index and all-cause mortality in US adults with and without diabetes. J Gen ntern Med. 2014;29(1):25-33.

2. Liao Q, Zheng Z, Xiu S, et al. Waist circumference is a better predictor of risk for frailty than BMI in the community-dwelling elderly in Beijing. Aging Clin Exp Res. 2018;30(11):1319-25. 
3. GBD 2017 Risk Factor Collaborators. Global, regional, and national comparative risk assessment of 84 behavioural, environmental and occupational, and metabolic risks or clusters of risks for 195 countries and territories, 1990-2017: a systematic analysis for the Global Burden of Disease Study 2017. Lancet. 2018;392(10159):1923-94.

4. Nicholas JA, Lo Siou G, Lynch BM, et al. Leisure-time physical activity does not attenuate the association between occupational sedentary behavior and obesity: results from Alberta's tomorrow project. J Phys Act Health. 2015;12(12):1589-600.

5. Shen C, Zhou Z, Lai S, et al. Urban-rural-specific trend in prevalence of general and central obesity, and association with hypertension in Chinese adults, aged 18-65 years. BMC Public Health. 2019;19(1):661.

6. Ouyang $\mathrm{Y}$, Wang $\mathrm{H}$, Su $\mathrm{C}$, et al. Use of quantile regression to investigate changes in the body mass index distribution of Chinese adults aged 18-60 years: a longitudinal study. BMC Public Health. 2015;15:278.

7. Rao DP, Patel P, Roberts KC, et al. Obesity and healthy aging: social, functional and mental well-being among older Canadians. Health Promot Chronic Dis Prev Can. 2018;38(12):437-44.

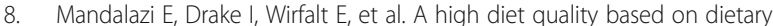
recommendations is not associated with lower incidence of type 2 diabetes in the malmö diet and cancer cohort. Int J Mol Sci. 2016;17(6):901.

9. Cohen JFW, Lehnerd ME, Houser RF, et al. Dietary approaches to stop hypertension diet, weight status, and blood pressure among children and adolescents: national health and nutrition examination surveys 2003-2012. J Acad Nutr Diet. 2017;117(9):1437-1444.e2.

10. Murphy KJ, Parletta N. Implementing a Mediterranean-style diet outside the Mediterranean region. Curr Atheroscleros Rep. 2018;20(6):28.

11. 11. Hu EA, Steffen LM, Coresh J, et al. Adherence to the healthy eating index-2015 and other dietary patterns may reduce risk of cardiovascular Disease, cardiovascular mortality, and all-cause mortality. J Nutr. 2020;150(2): $312-21$

12. Wang $X$, Liu A, Du M, et al. Diet quality is associated with reduced risk of hypertension among Inner Mongolia adults in northern China. Public Health Nutr. 2020;23(9):1543-54

13. Dugee $\mathrm{O}$, Khor GL, Lye MS, et al. Association of major dietary patterns with obesity risk among Mongolian men and women. Asia Pac J Clin Nutr. 2009; 18(3):433-40.

14. Li Y, Wang H, Wang KJ, et al. Optimal body fat percentage cut-off values for identifying cardiovascular risk factors in Mongolian and Han adults: a population-based cross-sectional study in Inner Mongolia. China BMJ Open. 2017;7(4):e014675

15. Zhao $Y$, Zhang M, Luo $X$, et al. Association of obesity categories and high blood pressure in a rural adult Chinese population. J Hum Hypertension. 2016;30(10):613-8.

16. Ng M, Fleming T, Robinson $M$, et al. Global, regional, and national prevalence of overweight and obesity in children and adults during 19802013: a systematic analysis for the global burden of disease study 2013. Lancet. 2014;384(9945):766-81.

17. Yang Y, Wang G, Pan X. China Food Composition. Beijing: Peking University Medical Press; 2009.

18. Fung TT, Chiuve SE, McCullough ML, et al. Adherence to a DASH-style die and risk of coronary heart disease and stroke in women. Arch Intern Med. 2008;168(7):713-20.

19. Fung $\Pi$, Rexrode KM, Mantzoros CS, et al. Mediterranean diet and incidence of and mortality from coronary heart disease and stroke in women. Circulation. 2009;119(8):1093-100.

20. Chinese Center For Disease Control And Prevention. Chinese adult overweight and obesity prevention and control guidelines. Beijing: People's Health Press; 2006

21. Li X, Jiang $Y$, Hu N, et al. Prevalence and characteristic of overweight and obesity among adults in China, 2010. Zhonghua Yu Fang Yi Xue Za Zhi. 2012;46:683-6.

22. Ramon-Arbues E, Martinez-Abadia B, Gracia-Tabuenca T, et al. Prevalence of overweight/obesity and its association with diabetes, hypertension, dyslipidemia and metabolic syndrome: a cross-sectional study of a sample of workers in Aragón, Spain. Nutr Hosp. 2019;36(1):51-9.

23. Chan YY, Lim KK, Lim KH, et al. Physical activity and overweight/obesity among Malaysian adults: findings from the 2015 National Health and morbidity survey (NHMS). BMC Public Health. 2017;17(1):733.

24. Marques A, Peralta M, Naia A, Loureiro N, et al. Prevalence of adult overweight and obesity in 20 European countries, 2014. Eur J Pub Health. 2018;28(2):295-300.
25. Muros JJ, Zabala M. Differences in Mediterranean diet adherence between Cyclists and Triathletes in a sample of Spanish Athletes. Nutrients. 2018; 10(10):1480

26. Bruno E, Manoukian S, Venturelli E, et al. Adherence to Mediterranean diet and metabolic syndrome in BRCA mutation carriers. Integr Cancer Ther. 2018;17(1):153-60.

27. Cheung LTF, Chan RSM, Ko GTC, et al. Diet quality is inversely associated with obesity in Chinese adults with type 2 diabetes. Nutr J. 2018;17(1):63.

28. Wang $C$, Lin $X L$, Fan $Y Y$, et al. Diet quality scores and risk of nasopharyngeal carcinoma in Chinese adults: a case-control study. Nutrients. 2016;8(3):112

29. Garcia M, Bihuniak JD, Shook J, Kenny A, et al. The effect of the traditional Mediterranean-style diet on metabolic risk factors: a meta-analysis. Nutrients. 2016:8(3):168.

30. Azadi-Yazdi M, Karimi-Zarchi M, Salehi-Abargouei A, et al. Effects of dietary approach to stop hypertension diet on androgens, antioxidant status and body composition in overweight and obese women with polycystic ovary syndrome: a randomised controlled trial. J Hum Nutr Diet. 2017:30(3):275-83.

31. Pavić E, Hadžiabdić MO, Mucalo I, et al. Effect of the Mediterranean diet in combination with exercise on metabolic syndrome parameters: 1-year randomized controlled trial. Int J Vitamin Nutr Res. 2019;89(3-4):132-43.

32. Church T, Martin CK. The obesity epidemic: a consequence of reduced energy expenditure and the uncoupling of energy intake? Obesity. 2018; 26(1):14-6.

33. Rouhani MH, Salehi-Abargouei A, Surkan PJ, et al. Is there a relationship between red or processed meat intake and obesity? A systematic review and meta-analysis of observational studies. Obes Rev. 2014;15(9):740-8.

34. Mullie P, Clarys P. Relation between dietary pattern analysis (principal component analysis) and body mass index: a 5-year follow-up study in a Belgian military population. J R Army Med Corps. 2016;162(1):23-9.

35. Wang Y, Beydoun MA. Meat consumption is associated with obesity and central obesity among US adults. Int J Obes. 2009;33(6):621-8.

36. Wang Z, Zhang B, Zhai $F$, et al. Fatty and lean red meat consumption in China: differential association with Chinese abdominal obesity. Nutr Metab Carbiovasc Dis. 2014:24(8):869-76.

37. Bluher M. Obesity: global epidemiology and pathogenesis. Nat Rev Endocrinol. 2019:15(5):288-98.

38. Aljuraiban GS, Chan Q, Oude Griep LM, et al. The impact of eating frequency and time of intake on nutrient quality and body mass index: the INTERMAP study, a population-based study. J Acad Nutr Diet. 2015;115(4): 528-36.

39. Cioffi I, Evangelista A, Ponzo V, et al. Intermittent versus continuous energy restriction on weight loss and cardiometabolic outcomes: a systematic review and meta-analysis of randomized controlled trials. J Transl Med. 2018;16(1):371

40. Dawczynski C, Kleber ME, März W, et al. Saturated fatty acids are not off the hook. Nutr Metab Carbiovasc Dis. 2015;25(12):1071-8.

41. Piers LS, Walker KZ, Stoney RM, et al. Substitution of saturated with monounsaturated fat in a 4-week diet affects body weight and composition of overweight and obese men. Br J Nutr. 2003:90(3):717-27.

42. Quennell JH, Howell CS, Roa J, et al. Leptin deficiency and diet-induced obesity reduce hypothalamic kisspeptin expression in mice. Endocrinology. 2011;152(4):1541-50

43. Folsom AR, Parker ED, Harnack $\amalg$. Degree of concordance with DASH diet guidelines and incidence of hypertension and fatal cardiovascular disease. Am J Hypertens. 2007:20(3):225-32.

44. Drouin-Chartier JP, Zheng Y, Li Y, et al. Changes in consumption of sugary beverages and artificially sweetened beverages and subsequent risk of type 2 diabetes: results from three large prospective U.S. cohorts of women and men. Diabetes Care. 2019;42(12):2181-9.

45. Malik VS. Sugar sweetened beverages and cardiometabolic health. Curr Opin Cardiol. 2017;32(5):572-9.

46. Zhong WW, Van Horn L, Cornelis MC, et al. Associations of dietary cholesterol or egg consumption with incident cardiovascular disease and mortality. JAMA. 2019:321(11):1081-95.

47. Magriplis E, Panagiotakos D, Kyrou I, et al. Presence of hypertension is reduced by Mediterranean diet adherence in all individuals with a more pronounced effect in the obese: the Hellenic National Nutrition and Health Survey (HNNHS). Nutrients. 2020;12(3):853.

48. Sánchez-Sánchez ML, García-Vigara A, Hidalgo-Mora JJ, et al. Mediterranean diet and health: a systematic review of epidemiological studies and intervention trials. Maturitas. 2020;136:25-37. 
49. Newman TM, Vitolins MZ, Cook KL. From the table to the tumor: the role of Mediterranean and Western dietary patterns in shifting microbial-mediated signaling to impact breast cancer risk. Nutrients. 2019;11(11):2565.

50. Hardman RJ, Kennedy G, Macpherson H, et al. Adherence to a

Mediterranean-style diet and effects on cognition in adults: a qualitative evaluation and systematic review of longitudinal and prospective trials. Front Nutr. 2016;3:22.

\section{Publisher's Note}

Springer Nature remains neutral with regard to jurisdictional claims in published maps and institutional affiliations.

- fast, convenient online submission

- thorough peer review by experienced researchers in your field

- rapid publication on acceptance

- support for research data, including large and complex data types

- gold Open Access which fosters wider collaboration and increased citations

- maximum visibility for your research: over $100 \mathrm{M}$ website views per year

At BMC, research is always in progress. 